\title{
A Fully Abstract Denotational Model for Higher-Order Processes (Extended Abstract)
}

\author{
M. Hennessy \\ CSAI \\ University of Sussex \\ Brighton BN1 9QH, UK
}

\begin{abstract}
A higher-order process calculus is defined in which one can describe processes which transmit as messages other processes; it may be viewed as a generalisation of the lazy $\lambda$-calculus. We present a denotational model for the language, obtained by generalising the domain equation for Abramskys model of the lazy $\lambda$-calculus. It is shown to be fully abstract with respect to three different behavioural preorders. The first is based on observing the ability of processes to perform an action in all contexts, the second on testing and the final one on satisfying certain kinds of modal formulae.
\end{abstract}

\section{Introduction}

The basic mathematical theory of process algebras is well-developed and fairly well understood, [Mil89, Hoa85, BW90, Hen88]. Much of this fundamental work has been carried out for "pure" process algebras, where the actions are taken to be simple synchronisation pulses along channels, but more recently theories have been developed for languages where various kinds of data are passed along the communication channels. For example in [Tho89, Tho90] processes may pass other processes as values; it is this type of process description language which is the topic of the present paper.

Here there are two kinds of action prefixing, $c$ ? X.P, meaning input a process along the channel $c$ and bind it to the process variable $X$ in the term $P$, and $c ! Q . P$, meaning output the process $Q$ along the channel $c$ and then act like the process $P$. Thus $c$ ? $X .(X \mid R)$ represents a process which can input any process and run it in parallel with $R$. Combining this with $c ! Q . P$ we obtain the process $c ! Q . P \mid c ? X .(X \mid R)$ which can perform a communication to become the process $P \mid(Q \mid R)$. This idea is pursued in depth in [Tho90] where a number of different formalisations are investigated. The resulting language is shown to be vel:y powerful in that it can simulate, in some sense, both the $\lambda$-calculus and the $\pi$-calculus of [MPW92]. The connection between the $\pi$-calculus and various highe:rorder process calculi and their relative merits is further pursued in [San92]. Here we do not address such issues. Rather we investigate the possibility of providing an adequate semantic theory for higher-order process calculi. In particular we provide a fully abstract denotational model for one such higher-order language.

The starting point for the development of this model is the lazy $\lambda$-calculus. At a very naive level this is a primitive higher-order process language. The $\lambda$-term $\lambda x . p$ may be viewed as a process which is waiting to receive another process along the communication channel $\lambda$ to be subsequently bound to $x$ in $p$ while the application term $p q$ represents sending the process $q$ to the process $p$. In [Abr90] this languagse is interpreted in the model obtained by solving the domain equation

$$
\begin{aligned}
& \mathbf{D}=\mathbf{F}_{\perp} \\
& \mathbf{F}=\mathbf{D} \longrightarrow \mathbf{D} .
\end{aligned}
$$

Each $\lambda$-term is interpreted either as $\perp$, in the case when it gives rise to a divergent computation, or as an element of $\mathbf{F}$, i.e. a function over $\lambda$-terms. A higher-order process can be viewed as having similar behaviour but now parametrised on channels, $\lambda$ terms being simple processes which can only receive input on one channel. Thus the input behaviour of a higher-order process, in analogy with $\lambda$-terms, can be captured by a function from $\mathcal{N}$, the set of challnel names, to $\mathbf{F}_{1}$; with respect to each channel the process may offer no behaviour, modelled by $\perp$, or may act like a function over processes. Similarly $i, s$ output behaviour, which has no real counterpart in the $\lambda$-calculus, can be modelled as a function from $\mathcal{N}$ 
to $\mathbf{C}_{\perp}$, where $\mathbf{C}$ is some space suitable for modelling output. One simple suggestion for $\mathbf{C}$ is the Cartesian product $\mathbf{D} \times \mathbf{D}$, with the elements of the pair representing, respectively, the process sent along the channel and the residual of the output action. It turns out that a slightly more complicated form of product is actually necessary, which we denote by $\mathbf{D} \otimes^{r} \mathbf{D}$.

The analogy between $\lambda$-terms and higher-order processes given above is rather tenuous but it has helped us motivate a model obtained by solving the recursive domain equation

$$
\begin{aligned}
& \mathbf{P}=\left(\mathcal{N} \longrightarrow \mathbf{C}_{\perp}\right) \times\left(\mathcal{N} \longrightarrow \mathbf{F}_{\perp}\right) \\
& \mathbf{F}=\mathbf{P} \longrightarrow \mathbf{P} \\
& \mathbf{C}=\mathbf{P} \otimes^{r} \mathbf{P}
\end{aligned}
$$

Moreover one can easily imagine how the combinators of input and output might be interpreted over this model. But a reasonable process algebra contains other combinators. At first glance the choice combinator + would appear to present problems. The $\lambda$ calculus is completely deterministic in its extensional behaviour but nondeterminism is an essential aspect of process algebras. However in [Bou91] it is shown how to interpret a nondeterministic version of the $\lambda$ calculus in the domain $D$; this is in fact a prime algebraic lattice and in particular it has a join operator $V$. The domain $\mathbf{P}$ is also a prime algebraic lattice and thus we can use exactly the same approach, interpreting the choice combinator + as $V$. Moreover having an interpretation of prefixing and choice means that using an expansion theorem we can obtain an interpretation of the parallel combinator $\mid$. Finally it turns out that we can also interpret in a straightforward fashion certain forms of channel scoping; that called dynamic scoping in [Tho90].

Having outlined a possible denotational semantics for a higher-order process calculus the next question we must address is: how reasonable is this as a model of the behaviour of processes? To answer this question we again pursue the analogy with the $\lambda$-calculus. How good is $\mathbf{D}$ as a model of the behaviour of $\lambda$-terms ? This question is answered in a very precise manner in [Abr90, Bou91] and we can give a very similar answer for $\mathbf{P}$.

In [Abr90] an observational preorder is defined on $\lambda$-terms in terms of their ability to converge to a "functional term" of the form $\lambda x . r$ using a lazy evaluation strategy. Let us denote this by $p \Downarrow$ and for two $\lambda$-terms $p, q$ let $p \prec q$ if $p \Downarrow$ implies $q \Downarrow$.

Let $p \check{\mathcal{O}}_{\mathcal{O}} q$ if for every context $C[]$, i.e. a term with one "hole" [] in it, $C[p] \prec C[q]$.

In [Abr90] it is shown that, subject to certain expressivity requirements, the domain $\mathbf{D}$ is fully abstra.ct with respect to the observational preorder $\check{\sim}_{\mathcal{O}}$. That is, $p \check{\sim}_{\mathcal{O}} q$ if and only if the interpretation of $p$ in the domain $\mathbf{D}$ is dominated by the interpretation of $q$; the domain properly reflects the ability of $\lambda$-terms to act like functions. A similar result holds for the the nondeterministic or parallel version of the $\lambda$-calculus of [Bou90, Bou91] where $p \Downarrow$ is interpreted as it is possible for $p$ to converge to a functional term, although in these papers a different phraseology is used.

Viewing the $\lambda$-calculus as a primitive higher-orcler process calculus $p \Downarrow$ can be interpreted as: $p$ is willing to offer a communication on the communication chennel $\lambda$. So let us generalise this predicate $\Downarrow$ to arbitrary processes from our higher-order process calculus by defining:

$p \Downarrow$ if there exists some channel on which $p$ is willing to offer a communication.

The main result of this paper is that, subject orice more to expressivity requirements, the model $\mathbf{P}$ is fully-abstract with respect to the observational preorder $\complement_{\mathcal{O}}$, with this new interpretation of $\Downarrow$. That is, the interpretation of the process $p$ in the domain $\mathbf{P}$ is dominated by that of $q$ if and only if for every context $C[]$ if $C[p]$ is willing to offer a communication on some channel then so is $C[q]$.

We also prove full abstraction for two other observational preorders between processes and both can also be motivated by reference to similar results for the lazy $\lambda$-calculus. The ability to examine a $\lambda$-term in an arbitrary context gives one complete control over that term; the context can for example send the ter:m to a collection of subterms each of which can examine an aspect of its behaviour and then pass it on to other subterms for further examination. However each of these subterms can only use the term under exarnination in a limited manner: they can only supply an argument for the term to be applied to. So a simpler behavioural preorder may be defined on $\lambda$ - terms based on their reaction to a sequence of arguments:

$$
\begin{aligned}
& p \check{\sim}_{\mathcal{T}} q \quad \text { if }\left(\ldots\left(p r_{1}\right) \ldots r_{n}\right) \Downarrow \text { implies } \\
& \left(\ldots\left(p r_{1}\right) \ldots r_{n}\right) \Downarrow \text { for every sequence of } \lambda \text { - } \\
& \text { terms } r_{1}, \ldots, r_{n} \text {. }
\end{aligned}
$$

The model $\mathbf{D}$ is also fully abstract with respect to this preorder, i.e. $\check{\Sigma}_{\mathcal{O}}$ and $\Xi_{\mathcal{T}}$ coincide over $\lambda$-terms. This view of $\lambda$-terms treats them as "black boxes". One has no control over them; the only way of finding 
out about their behaviour is to send them a parameter, i.e. communicate with them. This is very similar in spirit to the theory of testing for processes, originally presented in [DH84] and expounded at length in [Hen88]. There a test $e$ (represented as another process) is applied to a process $p$ by running $e$ and $p$ in parallel, thereby allowing them to communicate, and the application is successful if $e$ reaches some "successful" state. The test $e$ may be viewed as a generalisation of the sequence of parameters $r_{1}, \ldots r_{n}$ supplied to the $\lambda$-term and the successful state plays the role of $\Downarrow$. So let us generalise $\check{\Sigma}_{\mathcal{T}}$ to higher-order processes by saying $p$ may satisfy the test $e$ if there is a successful application of $e$ to $p$ and

$p \check{\sim}_{T} q$ if $p$ may satisfy $e$ implies $q$ may satisfy $e$ for every test $e$.

We show that $\mathbf{P}$ is also fully abstract with respect to $\check{\sim}_{\tau}$.

The full abstraction results in [Abr90, Bou91] rely heavily on a logical characterisation of the domain $\mathbf{D}$, [CC90, Abr91]. Essentially the compact elements of $D$ can be described by formulae, $\phi$, from a logic in such a way that $\mathbf{D}$ is isomorphic to the filters generated by the logic. Further the interpretation of the $\lambda$-calculus in $\mathbf{D}$ can be completely captured by a program logic whose judgements are of the form $\vdash p: \phi$ and this program logic is central to the proofs of full abstraction. A similar program can be carried out for the model $\mathbf{P}$ and the resulting logic is a simple modal logic whose formulae express the ability of processes to receive and transmit along communication channels. Furthermore we can interpret this modal language operationally over processes using a satisfaction relation $\models$ between processes and formulae; it is called a realizability interpretation in [Bou91]. This gives a further method for comparing behaviourally processes:

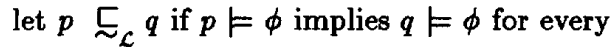
formulae $\phi$.

We also show that $\mathbf{P}$ is fully abstract with respect to $ᄃ_{f}$. This is analogous to the modal characterisation of bisimulation equivalence for processes given in [HM85].

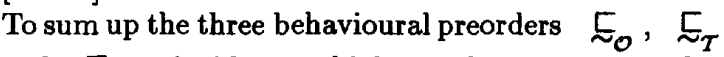
and $\check{\sim}_{\mathcal{L}}$ coincide over higher-order processes definable in our language and further they are characterised precisely by the model $\mathbf{P}$.

The literature on higher-order process algebras is rather limited. We have already refered to [Tho90], on whose language we base our algebra. Their relationship with the $\pi$-calculus is studied in [San9'2] while [AR87, GMP90, Nie89] present higher-order programming languages which contains among other constructs a sophisticated type structures for the values transmitted between processes. The addition of parallelism to the $\lambda$-calculus is studied extensively in [Bou90, Bou91, Bou92]; in particular fully-abstrait models, filter models of logics, are constructed for the observational preorder over parallel- $\lambda$-terms. Howevier all of this research concentrates on developing a bihavioural theory of these languages. The only denotational model for such languages of which the author is aware is presented in [JP90]; this is for a very simple subset of the $\gamma$-calculus of [Bou89], a mild generalisistion of the $\lambda$-calculus, and moreover the model is only shown to be adequate.

Most details of the proofs are omitted from this extended abstract. They may be found in the full version of the paper, [Hen92], which also contains a more leisurely account of the various concepts and techniques used.

\section{The Language}

The syntax of the language is given by

$\begin{array}{llll}\text { Processes } & T::= & N I L|T+T| n ? F \\ & & & |n ! C| X \mid F T \\ & & \mid G(T), G \in A u x \\ \text { Abstractions } & F & := & (X) T \\ \text { Concretions } & C & ::= & {[T] T}\end{array}$

where $n$ ranges over a set of channel names $\mathcal{N}, X$ ranges over a set of process variables $\mathcal{X}$ and $A u x$ is a set of auxiliary operators. In this paper we use a particular set of such operators: for each pair of subsets, $\mathcal{A}, \mathcal{B}$ of $\mathcal{N}$, a binary infix parallel operator ${ }_{A} \mid \mathcal{B}$ and for each function $r$ from $\mathcal{N}$ to $\mathcal{N}$ which is almoist everywhere the identity a unary postfix renaming operator $\{r\}$. In $(X) T$ the prefix $(X)$ acts as a binder for occurrences of $X$ in $T$ and this leads to the standard notion of free and bound occurrences of variables, $\alpha$-conversion and of substitution. We use process to mean a closed process-term from this language and $P, Q, \ldots$ are used to denote typical processes.

The operational semantics of the language is given in Figure 1 where for convenience we have omitted the symmetric counterparts to the Choice and Parallelism rules and the function name used in the latter has the obvious definition. There are three types of 
Input:

Output:

$$
n ? F \stackrel{n ?}{\longrightarrow} F
$$

Choice:

$$
n ! C \stackrel{n !}{\longrightarrow} C
$$

Application:

$$
P \stackrel{c}{\longrightarrow} A \text { implies } P+Q \stackrel{c}{\longrightarrow} A
$$

Parallelism

$$
\begin{aligned}
& T\{Q / X\} \stackrel{c}{\longrightarrow} A \text { implies } F Q \stackrel{c}{\longrightarrow} A \\
& \text { where } F \text { is }(X) T
\end{aligned}
$$

Communication

$$
\begin{aligned}
& P \stackrel{c}{\longrightarrow} A, \quad \text { name }(c) \in \mathcal{A} \cup\{r\} \text { implies } \\
& \left.P_{\mathcal{A} \mid \mathcal{B}} Q \stackrel{c}{\longrightarrow} A_{\mathcal{A}}\right|_{\mathcal{B}} Q
\end{aligned}
$$

$$
P \stackrel{n ?}{\longrightarrow} F, Q \stackrel{n !}{\longrightarrow}\left[Q_{1}\right] Q_{2} \text { implies }
$$

Renaming:

$$
\left.\left.P_{\mathcal{A}}\right|_{\mathcal{B}} Q \stackrel{T}{\longrightarrow} F Q_{1}\right|_{\mathcal{B}} Q_{2}
$$

$$
P \stackrel{c}{\longrightarrow} A \text { implies } P\{r\} \stackrel{r(c)}{\longrightarrow} A\{r\}
$$

where

if $F$ is $(X) T$ then $F A_{\mathcal{A}} P$ denotes $(X)\left(\left.T_{A}\right|_{\mathcal{B}} P\right)$ (and similarly for $\left.P_{A}\right|_{B} F$ ) and $F\{r\}$ denotes $(X)(T\{r\})$

if $C$ is $\left[Q_{1}\right] Q_{2}$ then $C_{\mathcal{A} \mid \mathcal{B}} P$ is $\left[Q_{1}\right]\left(\left.Q_{2 \mathcal{A}}\right|_{\mathcal{B}} P\right)$ (and similarly for $\left.P_{\mathcal{A}} \mid{ }_{\mathcal{B}} C\right)$ and $C\{r\}$ denotes $\left[Q_{1}\right]\left(Q_{2}\{r\}\right)$

Figure 1: The Operational semantics

judgements, of the form

$$
\begin{aligned}
& P \stackrel{n ?}{\longrightarrow} F \\
& P \stackrel{n !}{\longrightarrow} C \\
& P \stackrel{\tau}{\longrightarrow} Q
\end{aligned}
$$

where $P$ and $Q$ are processes, $F$ is a closed abstraction term and $C$ a closed concretion term. The relations $\stackrel{n ?}{\longrightarrow}$ and $\stackrel{n !}{\longrightarrow}$ describe the communication capabilities of processes while $\stackrel{\tau}{\longrightarrow}$ describes the affect of an actual communication; $P \stackrel{r}{\longrightarrow} Q$ means that $P$ may perform a communication and thereby be transformed into $Q$ and this may be considered as the counterpart to $\beta$ reduction in the $\lambda$-calculus.

With this operational semantics the language may be seen as a simple extension to CHOCS from [Tho90]. Channel restriction or hiding is implemented by the parameterised parallel operator; in $P A_{\mathcal{A}} Q$ all occurrences of names not from $\mathcal{A}$ in $P$ are local to $P$ and similarly for $\mathcal{B}$ in $Q$. With this view of restriction our operational semantics implements a dynamic scoping mechanism. To see this let us use the more usual notation $P \mid Q$ for $\left.P_{\mathcal{N}}\right|_{\mathcal{N}} Q$ and $P \backslash \mathcal{A}$ in place of $\left.P_{B}\right|_{\mathcal{N}} N ! L$ where $\mathcal{B}$ is $\mathcal{N}-\mathcal{A}$; this latter process acts like $P$ except that all channels in $\mathcal{A}$ are local. Now consider the term

$$
n ?(X)(X \mid P) \mid(n ![Q] R) \backslash \mathcal{A}
$$

where $Q$ is a process using some channels from $\mathcal{A}$. The occurrences of channels from $\mathcal{A}$ in $Q$ are governed by the restriction, i.e. they are local. Now a communication using the channel $n$ is possible and when it happens the process is transformed into

$$
(X)(X \mid P) Q \mid(R) \backslash \mathcal{A} .
$$

The result is that $Q$ has escaped from the restriction by being sent from one process to the other.

Based on this operational semantics we give three different behavioural equivalences or preorders. The first is motivated from the view of the lazy $\lambda$-calculus advocated in [Abr90, Bou90], as explained in the Introduction. To define $\check{\sim}_{\mathcal{O}}$ it is sufficient to define the convergence predicate $\Downarrow$ on processes:

$$
\begin{aligned}
& p \Downarrow \text { if there exists some } q \text { and channel } n \text { such } \\
& \text { that } p \stackrel{\tau}{\longrightarrow} * \stackrel{c}{\longrightarrow}, \text { for } c=n \text { ! or } c=n \text { ?. }
\end{aligned}
$$

The second behavioural preorder, $\Sigma_{\mathcal{T}}$, is a direct application of the general framework of testing, [Hen88], again as explained in the Introduction. Here the idea is that two processes are deemed to be equivalent unless there is a test or experiment which distinguishes between them. Processes are considered to be independent entities or "black boxes" and a test consists of a series of interactions between the process and the tester which continue until such time as the the tester reaches what it considers to be a successful state. The tester has no control over the process; it simply tries to communicate with the process, the process may deem to reply and if it does the tester may proceed with the experiment in a manner dependent on the reply of the process. In the present setting we take as a test any process which may use a new distinguished name $\omega$ and say it is in a successful state if it can perform the action $\omega !$. The application of the test $E$ to the process $P$ is defined to be a maximal sequence of the form

$E\left|P=E_{0}\right| P_{0} \stackrel{\tau}{\longrightarrow} E_{1}\left|P_{1} \stackrel{\tau}{\longrightarrow} \ldots \stackrel{\tau}{\longrightarrow} E_{k}\right| P_{k} \stackrel{r}{\longrightarrow} \ldots$ and it is a successful application if there exists an $n \geq$ 0 such that $E_{n} \stackrel{\omega !}{\longrightarrow}$. Then we say $P$ may $E$ if there exists an application of $E$ to $P$ which is successful. Finally 


\section{$P \check{\sim}_{\mathcal{T}} Q$ if for every test $E P$ may $E$ implies $Q$ may $E$.}

The third behavioural preorder is based on a "realizability" interpretation for the language using a generalised modal language $\mathcal{L}$. Formulae represent properties of processes and may indeed be an appropriate generalisation to concurrent systems of the notion of "type" commonly used for functional programming languages. For each of the syntactic categories, processes, abstractions and concretions, we have a corresponding set of formulae, $\mathcal{L}^{P}, \mathcal{L}^{F}, \mathcal{L}^{C}$ respectively. These are defined by

Processes:

$\omega \in \mathcal{L}^{P}$

$\phi \in \mathcal{L}^{F}$ implies $\langle n ?\rangle \phi \in \mathcal{L}^{P}$

$\phi \in \mathcal{L}^{C}$ implies $\langle n !\rangle \phi \in \mathcal{L}^{P}$

Abstractions:

$$
\phi_{1}, \ldots, \phi_{k}, \psi \in \mathcal{L}^{P} \text { implies } \phi_{1}, \ldots, \phi_{k} \rightarrow \psi \in \mathcal{L}^{F}
$$

Concretions:

$$
\phi_{1}, \ldots, \phi_{k}, \psi \in \mathcal{L}^{P} \text { implies }\left[\phi_{1}, \ldots, \phi_{k}\right] \psi \in \mathcal{L}^{C}
$$

The satisfaction relation, $\models^{\mathcal{O}}$, is also typed in that it is only defined between closed terms of each syntactic category and modal formulae from the corresponding language. It is defined as follows, where $P \stackrel{c}{\Longrightarrow} A$ is an abbreviation for $P \stackrel{\tau}{\longrightarrow}{ }^{*} P^{\prime} \stackrel{c}{\longrightarrow} A$. :

$$
\begin{aligned}
& P \models^{\mathcal{O}} \omega \text { for every process } P \\
& P \stackrel{n ?}{\Longrightarrow} F, F \models^{\mathcal{O}} \phi \text { implies } P \models^{\mathcal{O}}\langle n ?\rangle \phi \\
& P \stackrel{n !}{\Rightarrow} C, C \models^{\mathcal{O}} \phi \text { implies } P \models^{\mathcal{O}}\langle n !\rangle \phi \\
& \text { if }\left(\forall P, P \models^{\mathcal{O}} \phi \text { implies } F P \models^{\mathcal{O}} \psi\right) \text { then } \\
& \quad F \models^{\mathcal{O}} \underline{\phi} \rightarrow \\
& \text { if } Q_{1} \models^{\mathcal{O}} \underline{\phi} \text { and } Q_{2} \models^{\mathcal{O}} \psi \text { then }\left[Q_{1}\right] Q_{2} \models^{\mathcal{O}}[\underline{\phi}] \psi
\end{aligned}
$$

Note that $\models^{\mathcal{O}}$ depends entirely on the behaviour of terms and not on their structure.

$$
\text { Let } P \coprod_{\mathcal{L}} Q \text { if } P \vDash^{\mathcal{O}} \phi \text { implies } Q \models^{\mathcal{O}} \phi \text { for }
$$
every formula $\phi$.

We aim to show that the three behavioural preorders, $\check{\sim}_{\mathcal{O}}, \check{\tau}_{\mathcal{T}}$ and $\Xi_{\mathcal{L}}$ coincide. The modal language is the key to the proof as it provides a crucial link with a natural denotational semantics which can be shown to be fully-abstract with respect to each of these preorders.

\section{The Model}

We have already seen the domain equation for the model in the introduction but it remains to explain both the construction $\otimes^{r}$ and why it is necessary. In analogy with the view of the domain $D$ for the lazy $\lambda$-calculus in [Bou90] if domain for processes, $\mathbf{P}$, is an algebraic lattice then we can interpret the choice operator + as the binary join, But if we use as the domain of concretions $\mathbf{C}$ the Cartesian product $\mathbf{P} \times \mathbf{P}$ ? the join operation in $\mathbf{C}$ is defined pointwise; it satisfies the law:

$$
\left(x_{1}, y_{1}\right) \vee\left(x_{2}, y_{2}\right)=\left(x_{1} \vee x_{2}, y_{1} \vee y_{2}\right)
$$

In such a model the processes $m ![n !+k !] N I L$ and $m ![n !] N I L+m ![k !] N I L$ (where we use $l !$ as a shor;hand for the process $1 ![N I L] N I L$, whenever it makes sense) would be identified although one can easily find a test to distinguish them. It turns out that we require a product which satisfies the weaker law:

$$
\left(x, y_{1}\right) \vee\left(x, y_{2}\right)=\left(x, y_{1} \vee y_{2}\right)
$$

Such a product can be defined in the category of prime algebraic lattices with continuous functions. In this category we say $f$ is multilinear if it preserves $V$ in each of its arguments, i.e. $f\left(d_{1}, \ldots, d_{i} \vee d_{i}^{\prime}, \ldots d_{k}\right):=$ $f\left(d_{1}, \ldots, d_{i}, \ldots d_{k}\right) \vee f\left(d_{1}, \ldots, d_{i}^{\prime}, \ldots d_{k}\right)$, for each $i$, a function of two arguments is called rightlinear whenever it preserves join in its second argument, i.e. $f\left(x, y_{1} \vee y_{2}\right)=f\left(x, y_{1}\right) \vee f\left(x, y_{2}\right)$ and for functions of one argument we abbreviate "multilinear" to "linear". For any pair $D_{1}, D_{2}$, let $D_{1} \otimes^{r} D_{2}$ be the prime algebraic lattice which satisfies

1. there exists a rightlinear continuous injection

$$
i^{*}: D_{1} \times D_{2} \longmapsto D_{1} \otimes^{r} D_{2},
$$

2. for any rightlinear continuous $f: D_{1} \times D_{2} \mapsto$ $E$ there exists a unique continuous lineer

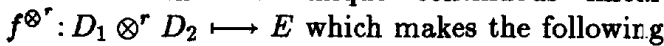
diagram commute:

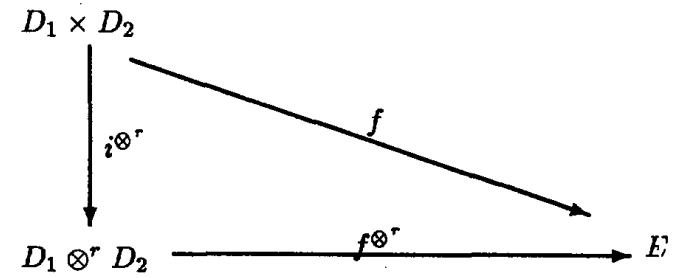

The construction of $D_{1} \otimes^{r} D_{2}$ is given in [Hen92] as is the proof that it is unique up to isomorphism. 
We us now briefly outline the interpretation of the language in this model. Most of the syntactic constructs are straightforward. Output on the channel $n$ is interpreted by the linear function $n_{\text {out }}: \mathbf{C} \longmapsto \mathbf{P}$ defined by

$n_{\text {out }}(c)=\left\langle\lambda x \in \mathcal{N} .\left(x=n \rightarrow c_{\perp}, \perp\right), \lambda x \in \mathcal{N} . \perp\right\rangle$

and input by the linear function $n_{i n}: \mathbf{F} \longmapsto \mathbf{P}$ defined in an analogous manner:

$n_{\text {in }}(f)=\left\langle\lambda x \in \mathcal{N} . \perp, \lambda x \in \mathcal{N} . x=n \rightarrow f_{\perp}, \perp>\right.$

Let $\sigma$ range over the set of environments, $E N V$, mappings from $\mathcal{X}$ to $\mathbf{P}$. Then the interpretation of the language is given by:

- $[N I L]_{P} \sigma=\perp$

- $[n ? F]_{P} \sigma=n_{i n}\left([F]_{F} \sigma\right)$

- $\left.[n ! C]_{P} \sigma=n_{\text {out }}(\llbracket C]_{C} \sigma\right)$

- $\left[X \rrbracket_{P} \sigma=\sigma(X)\right.$

- $[T+U]_{P} \sigma=[T]_{P} \sigma \vee[U]_{P} \sigma$

- $\left[F T \rrbracket_{P} \sigma=\llbracket F \rrbracket_{F} \sigma\left(\llbracket T \rrbracket_{P} \sigma\right)\right.$

- $[(X) T]_{F} \sigma=\lambda d \in \mathbf{P} \cdot[T]_{P} \sigma[X \mapsto d]$

- $\left[[T]_{C}\right]_{C} \sigma=[T]_{P} \sigma \otimes r[U]_{P} \sigma$

- $[G(\underline{T})]_{P} \sigma=g\left([T]_{P}\right)$

where for each auxiliary function symbol $G$ we have a corresponding multilinear function $g$ of the appropriate type. The details are given in the appendix.

Following [CC90, Sco82] we can also give a logical presentation of both the denotational model and the interpretation of the language. First we examine the model. Consider the logic in Figure 2. The judgements are of the form $\phi \leq \psi$ where $\phi$ is a non-empty vector of formulae and we assume that all the formulae $\phi_{i}$ and $\psi$ have the same type. Let us write $t \phi \leq \psi$ if $\phi \leq \psi$ can be derived using these inference rules and more generally $\vdash \phi \leq \psi$ if $\phi \leq \psi_{j}$ can be derived for each $j$. This logic characterises precisely to the model in the sense that each formulae $\phi \in \mathcal{L}^{A}$, for $A=P, F, C$, can be interpreted as an element $\llbracket \phi \rrbracket$ in A so that

- $[\phi]$ is prime

- every prime element is denoted by some formula, and therefore every compact element is denoted by some vector of formulae if we define $[\phi]$ to be $\llbracket \phi_{1} \rrbracket \vee \ldots \vee \llbracket \phi_{n} \rrbracket$

\section{General :}

Refl

$$
\phi \leq \phi
$$

Weak

$$
\frac{\phi \leq \psi}{\underline{\phi}, \phi^{\prime} \leq \psi}
$$

Trans

$$
\frac{\phi \leq \psi, \psi \leq \xi}{\underline{\phi} \leq \xi}
$$

Processes :

$$
\begin{array}{cc}
\mathcal{L} P_{1} & \phi \leq \omega \\
\mathcal{L} P_{2} & \frac{\phi}{\langle(c\rangle \phi} \leq\langle(c\rangle \psi
\end{array}
$$

Concretions :

$\mathcal{L} C_{1}$

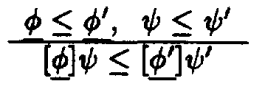

Figure 2: The proof system for $\mathcal{L}$

- $[\underline{\psi}] \leq[\underline{\phi}]$ if and only if $\vdash \underline{\phi} \leq \underline{\psi}$

This interpretation is given by:

Definition 3.1

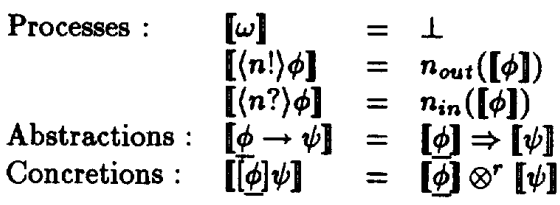

where $\Rightarrow$ represents the usual step function defined by

$$
d \Rightarrow e(x)= \begin{cases}e & d \leq x \\ \perp & \text { otherwise }\end{cases}
$$

This relationship between the model and the logic can be elegantly described in terms of filters. An A-filter 
is a non-empty subset $F$ of vectors of formulae from $\mathcal{L}^{A}$ with the property that $\phi \in F$ and $\mathcal{L} \vdash \phi \leq \underline{\psi}$ implies $\psi \in F$. Let $F i l^{A}$ be the set of all A-filters ordered by set inclusion.

Theorem 3.2 For $A=P, F, C, \quad F i l^{A}$ is a domain and isomorphic to $\mathbf{A}$.

The primes of the domain $\mathbf{P}$ are all denotable by formulae from $\mathcal{L}^{P}$ and the functions corresponding to the auxiliary operators, because they are multilinear, are determined by their effect on primes. So these functions can also be described in terms of logic; if $G$ is of arity $k$ we define a function $G:\left(\mathcal{L}^{p}\right)^{k} \longmapsto \mathcal{P}_{f}\left(\mathcal{L}^{p}\right)$, from vectors of formulae to finite sets of formulae, with the property that $g\left(\left[\phi_{1}\right], \ldots,\left[\phi_{k}\right]\right)=[G(\phi)]$; on the left hand side $g$ represents the semantic function applied to the elements $\llbracket \phi_{1} \rrbracket, \ldots, \llbracket \phi_{k} \rrbracket$ while on the right hand side $G$ is a function applied to a vector of formulae and the resulting finite set of formulae is interpreted in the obvious way using $V$.

The function $\{r\}$ over formulae is defined by the two clauses

$$
\begin{aligned}
& \text { - } \omega\{r\}=\omega \\
& \text { - }(\langle c\rangle(\underline{\phi}) \psi)\{r\}=r(c)(\underline{\phi}) \xi \text { where } \xi=\psi\{r\} .
\end{aligned}
$$

The main difficulty is in defining the parallel function $A \mid B$. This is given in tabular form in Figure 3, where some of the symmetric cases have been omitted. To make these definitions more compact we have used the notation $\langle c\rangle(\phi) \psi$ to mean $\langle n ?\rangle \phi \Rightarrow \psi$ when $c$ is $m$ ? and $\langle m !\rangle[\phi] \psi$ when it is $m !$.

Theorem $3.3 g\left(\llbracket \phi_{1} \rrbracket, \ldots, \llbracket \phi_{k} \rrbracket\right)=\llbracket G(\phi) \rrbracket$ for every $G$ in $A u x$.

Proof: By structural induction on $\phi$.

Next we show that the interpretation of the process language in the denotational model can also be captured in a logical form. We design a program logic whose judgements are of the form

$$
\Gamma \vdash^{a} A: \phi
$$

where $\phi \in \mathcal{L}^{A}$ for each $A=P, F, C$ and $\Gamma$ is an assumption. A assumption is a finite map from $\mathcal{X}$ to non-empty finite subsets of $\mathcal{L}^{P}$. We will actually represent these finite subsets as vectors of the form $\phi$. Let $\Gamma(X)$ denote $\omega$ whenever $X$ is not in the domain of $\Gamma$ and $\Gamma[X \mapsto \phi]$ represent the assumption obtained from $\Gamma$ in the obvious way - it coincides with $\Gamma$ for

$$
\begin{aligned}
& \alpha,\left.\beta \quad \alpha_{A}\right|_{B} \beta \\
& \omega, \omega \quad \omega \\
& \omega,\langle c\rangle(\phi) \psi \quad\{\omega\} \\
& \langle n ?\rangle \phi \rightarrow \psi \text {, } \\
& \langle m ?\rangle^{\prime} \underline{\phi}^{\prime} \rightarrow \psi^{\prime} \quad\{\omega\} \\
& \cup\left\{\langle n ?\rangle \underline{\phi} \rightarrow \xi\left|\xi \in \psi_{\mathcal{A}}\right|_{\mathcal{B}} \beta, n \in \mathcal{A}\right\} \\
& \cup\left\{\langle n ?\rangle \underline{\omega} \rightarrow \xi\left|\xi \in \omega_{\mathcal{A}}\right|_{\mathcal{B}} \beta, n \in \mathcal{A}\right\} \\
& \cup\left\{\langle m ?\rangle \Phi^{\prime} \rightarrow \xi\left|\xi \in \alpha_{\mathcal{A}}\right|_{\mathcal{B}} \psi^{\prime}, m \in \mathcal{B}\right\} \\
& \cup\left\{\langle m ?\rangle \underline{\underline{\omega}} \rightarrow \xi\left|\xi \in \alpha_{\mathcal{A}}\right|_{\mathcal{B}} \omega, m \in \mathcal{B}\right\} \\
& \cup\left\{(n !)[\phi] \xi\left|\xi \in \psi_{\mathcal{A}}\right|_{\mathcal{B}} \beta, n \in \mathcal{A}\right\} \\
& \cup\left\{\langle m !\rangle\left[\underline{\phi}^{\prime}\right] \xi\left|\xi \in \alpha_{\mathcal{A}}\right|_{\mathcal{B}} \psi^{\prime}, m \in B\right\} \\
& \cup\left\{\langle n ?\rangle \underline{\phi} \rightarrow \xi\left|\xi \in \psi_{\mathcal{A}}\right|_{\mathcal{B}} \beta, n \in \mathcal{A}\right\} \\
& \cup\left\{\langle n ?)_{\underline{\omega}} \rightarrow \xi\left|\xi \in \omega_{\mathcal{A}}\right|_{\mathcal{B}} \beta, n \in \mathcal{A}\right\} \\
& \cup\left\{\langle m !)\left[\phi^{\prime}\right] \xi\left|\xi \in \alpha_{\mathcal{A}}\right|_{\mathcal{B}} \psi^{\prime}, m \in \mathcal{B}\right\} \\
& \cup\left\{\xi\left|\xi \in \omega_{\mathcal{A}}\right|_{\mathcal{B}} \psi^{\prime}, m=n\right\} \\
& \cup\left\{\xi\left|\xi \in \psi_{\mathcal{A}}\right|_{\mathcal{B}} \psi^{\prime}, \mathcal{L} \vdash \underline{\phi}^{\prime} \leq \underline{\phi}, m:=n\right\}
\end{aligned}
$$

Figure 3: The parallel operator on formulae

every name except $X$ which it maps to $\phi$. We will also use $\Gamma \vdash^{a} A: \phi$ as an abbreviation for the $k$ judgements $\Gamma \vdash^{a} A: \bar{\phi}_{i}, 1 \leq i \leq k$ and by and large we omit the superscripts from consequence relations, rendering $\vdash^{a}$ as $\vdash$. The rules for deriving judgements are given in Figure 4. Note that the rule AuxR uses the interpretation of the auxiliary operators over the formulae. In order to establish that the process $G(\underline{T})$ satisfies a property $\psi$ in general it is necessary to establish that each $T_{i}$ satisfies $\phi_{i}$ for a vector of formulae $\phi$ such $\mathcal{L} \vdash G(\phi) \leq \psi$. This is not a very practical way to proceed and a more realistic program logic would replace the rule AuxR with individual rules which use the syntactic structure of the terms $G(\underline{T})$. However the present rule is adequate for our purposes.

This program logic can be interpreted over the denotational model in the following manner. For $\sigma \in E N V$ let $\sigma \vDash \Gamma$ if $\llbracket \Gamma(X) \rrbracket \leq \sigma(X)$ for all $X$. Then we write $\Gamma \models^{a} A: \phi$ if $\sigma \models \Gamma$ implies $\llbracket \phi \rrbracket \leq \llbracket A \rrbracket \sigma$.

Proposition 3.4 (Soundness) $\Gamma \vdash^{a} A: \phi$ implies 


\section{General :}

$\mathcal{L} R$

$\frac{\Gamma \vdash^{a} A: \phi, \quad \mathcal{L} \vdash \phi \leq \psi}{\Gamma \vdash^{a} A: \psi}$

Processes :

$\begin{array}{lc}\text { NR } & \Gamma[X \mapsto \phi] \vdash^{p} X: \phi_{i} \\ \omega R & \Gamma \vdash^{p} T: \omega \\ \text { PreR } & \frac{\Gamma \vdash^{f} F: \phi}{\Gamma \vdash^{p} n ?:\langle n ?\rangle \phi} \\ & \frac{\Gamma \vdash^{c} C: \phi}{\Gamma \vdash^{p} n ! C:\langle n !\rangle \phi} \\ & \frac{\Gamma \vdash^{p} T: \phi}{\Gamma \vdash^{p} T+U: \phi} \\ \text { JoinR } & \frac{\Gamma \vdash^{p} T: \phi}{\Gamma \vdash^{p} U+T: \phi} \\ \text { ApR } & \frac{\Gamma \vdash^{f} F: \phi \rightarrow \psi, \Gamma \vdash^{p} T: \phi}{\Gamma \vdash^{p} F T: \psi} \\ \text { AuxR } & \frac{\Gamma \vdash^{p} T_{i}: \phi_{i}, \mathcal{L} \vdash^{p} G(\phi) \leq \psi}{\Gamma \vdash^{p} G(\underline{T}): \psi}\end{array}$

Abstractions :

FunR

$$
\frac{\Gamma[X \mapsto \phi] \vdash^{p} T: \psi}{\Gamma \vdash^{\prime}(X) T: \underline{\phi} \rightarrow \psi}
$$

\section{Concretions :}

ConR

$$
\frac{\Gamma \vdash^{p} T: \phi, \quad \Gamma \vdash^{p} U: \psi}{\Gamma \vdash^{c}[T] U \cdot[\phi] \psi}
$$

Figure 4: The program logic

$$
\Gamma \vDash a: \phi
$$

Proof: It is sufficient to show that $\Gamma \models^{a} A: \phi$ satisfies the defining rules of $\Gamma \vdash^{a} A: \phi$. Note that the rule AuxR is justified by Theorem 3.3.

Theorem 3.5 (Completeness) $\Gamma \models^{a} A: \phi$ implies
$\Gamma \vdash^{a} A: \phi$

Proof: For any assumption $\Gamma$ let $\sigma_{\Gamma}$ be the environment defined by $\sigma_{\Gamma}(X)=\llbracket \Gamma(X) \rrbracket$. Then one can show by induction over $A$ that $[\phi] \leq[A] \sigma_{\Gamma}$ implies $\Gamma \vdash^{a} A: \phi$. The result now follows because $\sigma_{\Gamma} \vDash I^{\prime} \square$

We can also interpret the program logic using the realizability or observational interpretation of section two. Soundness is straightforward but the more difficult completeness will be given in the next section.

If $\rho$ is a closed substitution we write $\rho \models^{\mathcal{O}} \Gamma$ if for every $X \in \mathcal{X} \rho(X) \models^{\mathcal{O}} \Gamma(X)$ and $\Gamma \vDash \mathcal{O} A: \phi$ if $\rho \vDash \mathcal{O}^{\mathcal{O}} \Gamma$ implies $A \models \phi$. Note that $\Gamma \vDash{ }^{\mathcal{O}} A: \phi$ refers to the operational behaviour of $A$ while $\Gamma F^{a} A: \phi$ refers to its denotational interpretation.

Proposition 3.6 $\Gamma \vdash A: \phi$ implies $\Gamma \models^{\mathcal{O}} A: \phi$.

Proof: Again it is sufficient to prove that the relation $\Gamma \models^{\mathcal{O}} A: \phi$ satisfies all the defining rules of $\Gamma \vdash A: \phi$.

\section{Full Abstraction}

In this section we prove the main results of the paper, connecting the denotational interpretation with the various behavioural preorders.

First some notation. Each element $\boldsymbol{p}$ of $\mathbf{P}$ may be considered as a pair of functions which we denote by $p$ ! and $p$ ? respectively. Moreover for each $n$ in $\mathcal{N}^{\prime}$ it will be convenient to denote $p_{!}(n)$ and $p_{?}(n)$ as $p(n !)$ and $p(n$ ?) respectively. The first lemma shows that the denotational interpretation is consistent with the operational semantics.

Lemma 4.1 1. For $c$ of the form $m$ ? or $m !, P \stackrel{c}{\longrightarrow}$ $A$ implies $\llbracket A \rrbracket \perp \leq \llbracket P \rrbracket(c)$

2. $P \stackrel{\tau}{\longrightarrow} Q$ implies $\llbracket Q \rrbracket \leq \llbracket P \rrbracket$.

Proof: Both statements are proved simultaneously by induction on the length of the derivation of the operational judgements.

As an immediate corollary we have the following andequacy result: 
Theorem 4.2 (Adequacy) $P \Downarrow$ if and only if $[P] \neq$ $\perp$.

Proof: First suppose that $P \Downarrow$, i.e. $P \stackrel{\tau}{\longrightarrow} * P^{\prime} \stackrel{c}{\longrightarrow}$ for some $c$. The first part of the previous lemma implies $\llbracket P^{\prime} \rrbracket(c) \neq \perp$ while the second part implies $[P] \leq \llbracket P \rrbracket$, i.e. $\llbracket P \rrbracket(c) \neq \perp$ and therefore that $[P] \neq \perp$.

Conversely suppose $\llbracket P \rrbracket \neq \perp$, i.e. for some $c,[P](c) \neq \perp$. Then by the completeness theorem for the program logic, Theorem 3.5, $\vdash P:\langle c\rangle \omega$. By the soundness of the program logic with respect to the realizability interpretation, Proposition $3.6, \vDash^{\mathcal{O}}$ $P:\langle c\rangle \omega$, i.e. $P \stackrel{c}{\Longrightarrow}$.

Note that we have in fact established the slightly stronger result: $P \stackrel{c}{\Longrightarrow}$ if and only if $\llbracket P \rrbracket(c) \neq 1$

As an immediate corollary we have one direction of the full abstraction result:

Corollary 4.3 For closed terms $[P \rrbracket \leq \llbracket Q \rrbracket$ implies $P \check{\sim}_{\mathcal{O}} Q$

Proof: Let $C[\mathrm{]}$ be any closed context such that $C[P] \stackrel{c}{\Longrightarrow}$. We must show that $C[Q] \stackrel{c}{\Rightarrow}$ which, by the adequacy result is equivalent to showing $\llbracket C[Q] \rrbracket(c) \neq$ $\perp$. But $\llbracket P \rrbracket \leq \llbracket Q \rrbracket$ implies $\llbracket C[P] \rrbracket \leq \llbracket C[Q] \rrbracket$ and another application of the adequacy result gives $\llbracket C[P] \rrbracket(c) \neq \perp$. It therefore follows that $[C[Q] \rrbracket(c) \neq$ $\perp$.

To prove the converse we need a a definability result.

Theorem 4.4 (Definability) For every $n, i \in \mathcal{N}$ and $\phi \in \mathcal{L}^{p}$ there exists a closed process term $P_{\phi}^{n, i}$ and a closed abstraction term $F_{\phi}^{n, i}$ of the form $(X)\left(T_{\phi}^{n, i}\{n\} \mid\right.$ $X)$, where $T_{\phi}^{n, i}$ is closed, such that for any $n, i$ not occurring in $\phi$

1. for all $d \in \mathbf{P}, \llbracket n ! \rrbracket \leq \llbracket F_{\phi}^{n, i} \rrbracket d$ if and only if $\llbracket \phi \rrbracket \leq d$

2. $\left[P_{\phi}^{n, i} \rrbracket=\llbracket \phi\right]$.

Proof: For convenience we use $T \nabla^{n} U$ to denote the term $\left.T_{0}\right|_{\mathcal{N}-\{n\}} n ?(Y) U$ where $Y$ is some variable not occurring in $U$. First suppose we have defined $F_{\phi_{i}}^{n, i}$, where $\underline{\phi}=\phi_{1} \ldots \phi_{k}$ Then we let $F_{\phi}^{n, i}$ denote the term

$$
(X)\left(\left(\left.T_{\phi_{1}\{n\}}^{n, i}\right|_{\bullet} X\right)[n \rightarrow i] \triangleright^{n}\left(\ldots\left(T_{\phi_{k}}^{n, i}\{n\} \mid \diamond X\right) \ldots\right)\right)
$$

where $[n \rightarrow i]$ denotes the renaming which renames $n$ to $i$ and leaves all other names untouched. In a similar vein if $P_{\phi}^{n, i}$ have been defined we let $P_{\phi}^{n, i}$ denote $P_{\phi_{1}}^{n, i}+$ $\ldots+P_{\phi_{k}}^{n, i}$ and trivially $\left[P_{\phi}^{n, i}\right]=\left[P_{\phi_{1}}^{n, i}\right] \vee \ldots \vee\left[P_{\phi_{k}}^{n, i}\right]$ The definitions of the required terms are by induction on the structure of $\phi$ :

$$
\begin{aligned}
& \omega \\
& P_{\phi}^{n, i}=\perp \text { and } F_{\phi}^{n, i}=(X)(n !\{n\} \mid \phi) \\
& \text { - }\langle m ?\rangle \phi \rightarrow \psi \\
& F_{\phi}^{n, i}=(X)\left(\left.m !\left[P_{\underline{\psi}}^{n, i}\right] T_{\phi}^{n, i}\{n\}\right|_{\phi} X\right) \text { and } P_{\phi}^{n, i}= \\
& m ?(X)\left(F_{\phi}^{n, i} X \triangleright^{n} \bar{P}_{\psi}^{n, i}\right) \\
& \text { - }\langle m !\rangle[\phi] \psi \\
& F_{\phi}^{n, i}=(X)\left(m ?(X)\left(F_{\phi}^{n, i} X[n \rightarrow i] \nabla^{n} T_{\psi}^{n, i}\right)_{[n]}\right. \\
& X) \text { and } P_{\phi}^{n, i}=m !\left[P_{\phi}^{n, i}\right] P_{\psi}^{n, i} \text {. }
\end{aligned}
$$

The proof that these terms have the required properties is carried out by structural induction on formulise. It depends on the fact that $\left[F_{\phi}^{n, i}\right] d$ is either $\perp$ or $[n !]$. for every $d$.

Theorem 4.4, together with adequacy, gives a converse:

Proposition 4.5 For closed terms $P \check{\sim}_{T} Q$ implies $\llbracket P \rrbracket \leq \llbracket Q \rrbracket$.

Proof: To prove $\llbracket P \rrbracket \leq \llbracket Q \rrbracket$ it is sufficient to prove $[\phi \rrbracket \leq \llbracket Q \rrbracket$ for any $\phi$ such that $\llbracket \phi \rrbracket \leq[P]$. For such a $\phi$ the definability theorem implies that $\left.[n !] \leq \llbracket F_{\phi}^{n, i}\right][P]$, for all $n, i$ not appearing in $\phi$, which can be rewritten as $\left[F_{\phi}^{n, i} \mathbb{[}[P](n !) \neq \perp\right.$. By the adequacy theorem this implies $F_{\phi}^{n, i} P \stackrel{n !}{\Longrightarrow}$. In general $F_{\phi}^{n, i} R \stackrel{n !}{\Longrightarrow}$ if and only

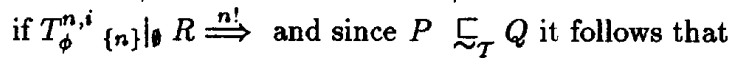
$F_{\phi}^{n, i} Q \stackrel{n !}{\Longrightarrow}$ which, again by the adequacy theorem, implies $\llbracket n ! \rrbracket \leq \llbracket F_{\phi}^{n, i} \rrbracket \llbracket Q \rrbracket$. Employing the definability result once more we obtain $\llbracket \phi \rrbracket \leq \llbracket Q \rrbracket$.

As an immediate corollary we have

Corollary 4.6 (Full Abstraction) For closed terins $P \check{\sim}_{\mathcal{O}} Q$ if and only if $P \check{\sim}_{\mathcal{T}} Q$ if and only if $P \check{\mathcal{L}}_{\mathcal{L}} Q$ if and only if $\llbracket P \rrbracket \leq \llbracket Q \rrbracket$.

Proof: One can easily establish that $\check{\sim}_{\mathcal{O}}$ is contained in $\complement_{\mathcal{T}}$ and therefore Corollary 4.3 and Proposition 4.5 establish that the both of these coincide with the preorder generated by the model. Because 
of Proposition 3.6 it is therefore sufficient to show $P \vDash \mathcal{O} \phi$ implies $\vdash P: \phi$. By the completeness result Theorem 3.5 this is equivalent to showing $P \vDash \mathcal{O} \phi$ implies $\left[\phi \rrbracket \leq \llbracket P \rrbracket\right.$. The more general result $A \vDash{ }^{\mathcal{O}} \phi$ implies $\llbracket \phi \rrbracket \leq \llbracket A \rrbracket$ for $A$ any closed process, abstraction or concretion term, can be established by induction on $\phi$; the case when $\phi$ has the form $\langle c\rangle \psi$ uses Lemma 4.1 while the case $\phi \rightarrow \psi$ depends on the fact that $\llbracket P_{\phi}^{n . i} \rrbracket=\llbracket \phi \rrbracket$ for any $n, i$ not occurring in $\underline{\phi}$ and therefore, by Proposition 3.6, that $P_{\Phi}^{n . i} \models^{\mathcal{O}} \phi$.

\section{Conclusions}

We have presented a semantic model of higherorder processes and shown it to be fully abstract with respect to a number of observational preorders. But these results raise many questions, some quite specific about our technical development and others more general.

It has been shown in [San92] that higher-order process languages can be simulated in the $\pi$-calculus but this is not to say that such languages are superfluous. They may provide convenient specification formalisms at an appropriate level of abstraction for describing the behaviour of sophisticated systems such as distributed operating or control systems, [LB92]. If this is the case then what kind of combinators should such a language have and can we model them using this semantic domain? Another question concerns the channel scoping mechanism used in the language. As we have seen $\mathbf{P}$ is adequate for modelling dynamic scoping but it has been argued in [Tho90] that static scoping of channels leads to a more natural language. At the moment it is not clear how to amend the definition of $\mathbf{P}$ so as to correctly model static scoping.

The program logic presented in section 6 provides the theoretical basis for a proof system for deriving properties of higher-order processes. As already pointed out this logic is not very realistic as to prove a parallel process $\left.p_{\mathcal{A}}\right|_{\mathcal{B}} q$ has a property $\psi$ it is necessary to find two formulae $\phi_{1}, \phi_{2}$ such that we can prove that $p$ has the property $\phi_{1}$ and $q \phi_{2}$ and then prove that the characteristic formula $\left.\phi_{1} A\right|_{B} \phi_{2}$ logically implies $\psi$. However this rule $A u x R$ could, at least in the case of the parallel operator, be replaced by more useful or easily applicable rules. The exact form these replacement rules should take remains to be seen.

Another line of possible future research concerns the behavioural preorders being modelled. That stud- ied in the present paper is very weak, at least ccmpared to many preorders defined for first-order process calculi. For such calculi it essentially corresponds to trace inclusion: $P \leq Q$ if every sequence of actions which $P$ can perform can also be performed by $Q$. This is very weak as it does not take into consideration the possible deadlocks or divergences of processes. For example it does not distinguish between the process $a . P$ and $a . P+a . N I L$ or $a . P+a . \Omega$ where $\Omega$ represents some process which can only perform an infinite internal computation. There are a large number of more discriminating behavioural preorders and equivalences in the extensive literature on process algebras, for example bisimulation equivalence [Mil89], failures equivalence [Hoa85] and the testing preorders of [Hen88]. These may easily be extended to higher-order processes and the approach to behavioural preorders in the definition of $\check{\leftarrow}_{\mathcal{O}}$ can also be strengthened so as to include information on deadlock. The simplest modification is to base the beisic comparison $\prec$ between processes not on their ability to perform actions but on their ability to converge: $P \Downarrow$ if there is no infinite internal computation from $P, P \stackrel{\tau}{\longrightarrow} P_{i} \stackrel{\tau}{\longrightarrow} \ldots \stackrel{\tau}{\longrightarrow}$ and $P$ may $\Downarrow$ if there exists some $Q$ such that $P \stackrel{\varepsilon}{\Rightarrow} Q$ and $Q \Downarrow$. The bisic comparison could now be defined by

$$
P \prec Q \text { if } P \text { may } \Downarrow \text { implies } P \text { may } \Downarrow \text {. }
$$

The resulting behavioural preorder is different than that which we have studied as it differentiates $N I L$ from $\Omega$ whereas they are identified in our theory. An even stronger comparison could be defined by

$$
P \prec Q \text { if } P \Downarrow \text { implies } Q \Downarrow \text {. }
$$

This leads to a behavioural theory which in general differentiates between processes of the form $a . P, a . P+$ $a . N I L$ and $a . P+a . \Omega$. It remains to be seen if fully abstract denotational models can be constructed for these theories.

\section{Appendix: The auxiliary functions}

To interpret the auxiliary functions over the domin $\mathbf{P}$ it is convenient to introduce some notational conventions. The first concerns the "lifting" operation. Suppose $t(\underline{x})$ is a meta-expression involving the variables $\underline{x}$ with the property that $t(\underline{v}) \in E$ for all values $v_{i}$ from a set $E^{i}$. Then if $w_{i} \in E_{\perp}^{i}, t(\underline{w})$ denotes the value in $E_{\perp}$ determined by

$$
t(\underline{w})= \begin{cases}\perp & \text { if } \exists i . w_{i}=\perp \\ t(\underline{v}) & \text { otherwise where } w_{i}=\left(v_{i}\right)_{\perp}\end{cases}
$$


The second convention is a convenient way of describing functions over tensor products. Let $\lambda\left(d_{1}, d_{2}\right) \in$ $D_{1} \times D_{2} . t$ represent a rightlinear function in $\left[D_{1} \times\right.$ $\left.D_{2} \longrightarrow E\right]$. Then we use $\lambda^{\otimes^{r}}\left(d_{1}, d_{2}\right) \in D_{1} \times D_{2}$.t to represent its unique extension to a linear function in $\left[D_{1} \otimes^{r} D_{2} \longrightarrow E\right]$.

The most difficult function to define is that corresponding to the parallel operator $\left.\mathcal{A}\right|_{\mathfrak{B}}$. Informally the definition simply mimics the usual interleaving interpretation of parallelism. Formally it takes the form $Y \operatorname{Par}_{\mathcal{A}, \mathcal{B}}$ where $Y$ is the least fixpoint operator and $\operatorname{Par}_{\mathcal{A}, \mathcal{B}}$ is a function of type $[\mathbf{P} \times \mathbf{P} \longrightarrow \mathbf{P}] \longrightarrow$ $[\mathbf{P} \times \mathbf{P} \longrightarrow \mathbf{P}]$. Intuitively if $F$ is of type $\mathbf{P} \times \mathbf{P} \longrightarrow \mathbf{P}$ then $\operatorname{Par}_{\mathcal{A}, \mathcal{B}} F$, when applied to two processes $x$ and $y$ calculates the resulting process by "unioning" together three different components. The first considers possible moves from $x$ and calculates their residuals by applying $F$ recursively, the second does the same for $y$ while the third calculates the possible results of communication between $x$ and $y$ using any channel in $\mathcal{N}$. Formally $\operatorname{Par}_{\mathcal{A}, \mathcal{B}} F(x, y)$ is defined by

$$
\begin{array}{cl}
\bigvee_{m \in \mathcal{A}} \quad & m_{\text {in }} \lambda d \in \mathbf{P} . F(x(m ?) d, y) \\
& \vee m_{\text {out }} C l(m) \\
\vee & \\
V_{m \in \mathcal{B}} \quad & m_{\text {in }} \lambda d \in \mathbf{P} . F(x, y(m ?) d) \\
& \vee m_{\text {out }} C r(m) \\
\vee & \\
V_{m \in \mathcal{N}} & \left(\lambda^{\otimes^{r}}\left(d, d^{\prime}\right) \in \mathbf{P} \times \mathbf{P} . F\left(x(m ?) d, d^{\prime}\right)\right) y(m !) \\
& \vee\left(\lambda^{\otimes^{r}}\left(d, d^{\prime}\right) \in \mathbf{P} \times \mathbf{P} . F\left(d^{\prime}, y(m ?) d\right)\right) x(m !)
\end{array}
$$

where

$$
\begin{aligned}
& C l(m)=\left(\lambda^{\otimes^{r}}\left(d, d^{\prime}\right) \in D \times D . d \otimes^{r} F\left(d^{\prime}, y\right)\right) x(m !) \\
& C r(m)=\left(\lambda^{\otimes^{r}}\left(d, d^{\prime}\right) \in \mathbf{P} \times \mathbf{P} . F\left(x, d^{\prime}\right) \otimes^{r} d\right) y(m !)
\end{aligned}
$$

The other auxiliary function, renaming, is handled in a similar manner. It is interpreted as $Y \operatorname{Ren}_{r}$ where for each renaming $r \quad \operatorname{Ren}_{r}:[\mathbf{P} \longrightarrow \mathbf{P}] \longmapsto[\mathbf{P} \longrightarrow \mathbf{P}]$ is defined by

$$
\begin{aligned}
\operatorname{Ren}_{r} F x= & \bigvee_{m \in \mathcal{N}} r(m)_{\text {in }} \lambda d \in \mathbf{P} \cdot F(x(m ?) d) \\
& \vee r(m !)_{\text {out }} C(m)
\end{aligned}
$$

where

$$
C(m)=\left(\lambda^{\otimes^{r}}\left(d, d^{\prime}\right) \in \mathbf{P} \times \mathbf{P} . d \otimes^{r} F\left(d^{\prime}\right)\right) x(m !)
$$

Acknowledgements: Thanks to Gerard Boudol for his detailed comments on a first draft of this paper.
This work has been supported by the SERC grant GR/H16537

\section{References}

[Abr90] S. Abramsky. The lazy lambda calculus. In D. Turner, editor, Research Topics in Functional Programming, pages 65-11i. Addison-Wesley, 1990.

[Abr91] Samson Abramsky. Domain theory in logical form. Ann. Pure Appl. Logic, 51:1-7?', 1991.

[AR87] E. Astesiano and G. Reggio. SMoLSdriven concurrent calculi. In TAPSOFT 1987, Lecture Notes in Computer Science 351, Lecture Notes in Computer Science, pages 169-201, 1987.

[Bou89] G. Boudol. Towards a lambda-calculus for concurrent and communicating systems. In J. Diaz, editor, Proc. TAPSOFT 89, pages 149-161. Springer-Verlag, 1989. LNCS 35L.

[Bou90] G. Boudol. A lambda-calculus for paralle] functions. Technical Report 1231, INRIASophia Antipolis, 1990.

[Bou91] G. Boudol. Lambda-calculi for (strict) parallel functions. Technical Report 1387, INRIA-Sophia Antipolis, 1991. To appear in Information and Computation.

[Bou92] G. Boudol. A calculus of communicating objects, 1992. To appear as INRIA Rzsearch Report.

[BW90] J. Baeton and W. Weijland. Process Algebra, volume 18 of Cambridge Tracts in Computer Scitence. Cambridge University Press, 1990.

[CC90] F. Cardone and M. Coppo. Two extensions of curry's type inference system. In P. Odifreddi, editor, Logic in Computior Science, pages 19-25. Academic Press, 1990.

[DH84] R. DeNicola and M. Hennessy. Testing equivalences for processes. Theoretical Computer Science, 24:83-113, 1984. 
[GMP90] A. Giacalone, P. Mistra, and S. Prasad. Operational and algebraic semantics for facile: A symmetric integration of concurrent and functional programming. In Proceedings of ICALP 90, volume 443 of Lecture Notes in Computer Science, pages 765-780, 1990.

[Hen88] M. Hennessy. An Algebraic Theory of Processes. MIT Press, 1988.

[Hen92] M. Hennessy. A fully abstract denotational model for higher-order processes. Technical Report 6/92, CSAI, University of Sussex, 1992.

[HM85] M. Hennessy and R. Milner. Algebraic laws for nondeterminism and concurrency. Journal of the Association for Computing Machinery, 32(1):137-161, 1985.

[Hoa85] C.A.R. Hoare. Communicating Sequential Processes. Prentice-Hall, 1985.

[JP90] R. Jagadeesan and P. Panangaden. A domain-theoretic model for a higher-order process calculus. In M.S.Paterson, editor, Proc. ICALP 90, pages 181-194. SpringerVerlag, 1990. LNCS 443.

[LB92] L. Leth and B.Thomsen. Some facile chemistry. Technical Report ERCC-92-14, ERCC, 1992.

[Mil89] R. Milner. Communication and Concurrency. Prentice-Hall, 1989.

[MPW92] R. Milner, J. Parrow, and D. Walker. A calculus of mobile proceses, part i. Information and Computation, 100(1):1-40, 1992.

[Nie89] F. Nielson. The typed $\lambda$-calculus with firstclass processes. In Proceedings of Parle 89, volume 366 of Lecture Notes in Computer Science, 1989.

[San92] D. Sangiorgo. Expressing Mobility in Process Algebras: First-Order and HigherOrder Paradigms. Phd thesis, Edinburgh University, Scotland, 1992.

[Sco82] D. S. Scott. Domains for denotational semantics. In M. Neilsen and E. M. Schmidt, editors, Proc. ICALP 82, pages 577-613. Springer-Verlag, 1982. LNCS 140.
[Tho89] B. Thomsen. A calculus of higher order communicating systems. In Conference Record of the Sixteenth Annual ACM Symposium on Principles of Programming Languages, pages 143-154, 1989.

[Tho90] B. Thomsen. Calculi for Higher-Onier Communicating Systems. Phd thesis, Imperial College, 1990. 